\title{
Application of the BHQ benzannulation reaction to the synthesis of benzo-fused coumarins
}

\author{
James A. Bull ${ }^{a}$, Cristina Luján ${ }^{a}$, Michael G. Hutchings ${ }^{b}$, Peter Quayle ${ }^{\mathrm{a}, *}$ \\ a School of Chemistry, The University of Manchester, Manchester M13 9PL, UK \\ ${ }^{\mathrm{b}}$ DyStar UK Ltd, School of Chemistry, The University of Manchester, Manchester M13 9PL, UK
}

\section{A R T I C L E I N F O}

\section{Article history:}

Received 13 January 2009

Revised 9 March 2009

Accepted 11 March 2009

Available online 16 March 2009

\section{Keywords:}

ATRC

Kharasch

Cyclisation

Radical

Copper

Carbene

Benzannulation

Lactone

\begin{abstract}
A B S T R A C T
A new approach to the synthesis of the $6 H$-benzo[d]naphtha[1,2-b]pyran-6-one ring system present in the gilvocarcin family of antibiotics is described. The key feature of this approach is the application of a new benzannulation strategy (the 'BHQ Reaction') whereby readily available ortho-allylaryl trichloroacetates are transformed into naphthalene derivatives via a cascade of reactions involving an initial ATRC reaction followed by the extrusion of $\mathrm{CO}_{2}$.
\end{abstract}

(C) 2009 Elsevier Ltd. All rights reserved.
During our investigations into the use of Atom Transfer Radical Cyclisation (ATRC) reactions in organic synthesis ${ }^{1}$ we recently happened upon a new synthesis of naphthalene derivatives ${ }^{2}$ starting from ortho-allylaryl trichloroacetates-the 'BHQ Reaction' (Scheme 1).

This unanticipated cyclisation sequence is compatible with a wide range of common functional groups and can be promoted using a variety of transition metal catalysts ${ }^{3}$ under either microwave activation conditions ${ }^{1}$ or purely thermal conditions. ${ }^{4}$ of the catalysts screened so far those that are copper based, most notably using a combination of $\mathbf{1}$ with $\mathrm{CuCl}$ or the preformed NHC carbene complex $\mathbf{2}^{5}$, appeared to be particularly effective in promoting this particular transformation.

Mindful of the fact that there exist relatively few general approaches ${ }^{6}$ to the synthesis of carbocyclic aromatics from simple acyclic precursors we have sought to define the scope and limitations of this methodology and herein we present its application to the synthesis of benzo-fused coumarin derivatives, a structural motif common to a variety of natural products. In particular we were interested in developing a general approach to the synthesis of the $6 H$-benzo[d]naphtha[1,2-b]pyran-6-one ring system ${ }^{7}$ which is common to a number of natural products such as gilvocarcin $\mathrm{V}^{8 a}$ and arnottin $\mathrm{I}^{\mathrm{Bb}}$ (Fig. 1).

\footnotetext{
* Corresponding author. Tel.: +44 161275 4619; fax: +44 1612754598

E-mail address: peter.quayle@manchester.ac.uk (P. Quayle).
}

The starting point of this study centred upon an investigation into the benzannulation reactions of trichloroacetates $\mathbf{5}$ and $\mathbf{8}$, substrates that were readily prepared in a three-step sequence from either 6-hydroxycoumarin 3 or umbelliferone (Scheme 2).

Conversion of $\mathbf{3}$ or umbelliferone to their respective allyl ethers followed by a thermally driven Claisen rearrangement (thermolysis in either $\mathrm{Ph}_{2} \mathrm{O}$ or $\mathrm{N}, \mathrm{N}$-diethylaniline) resulted in the isolation of phenols $\mathbf{4}^{9}$ and $\mathbf{7}^{10}$ in good overall yield. Acylation of $\mathbf{4}$ and $\mathbf{7}$
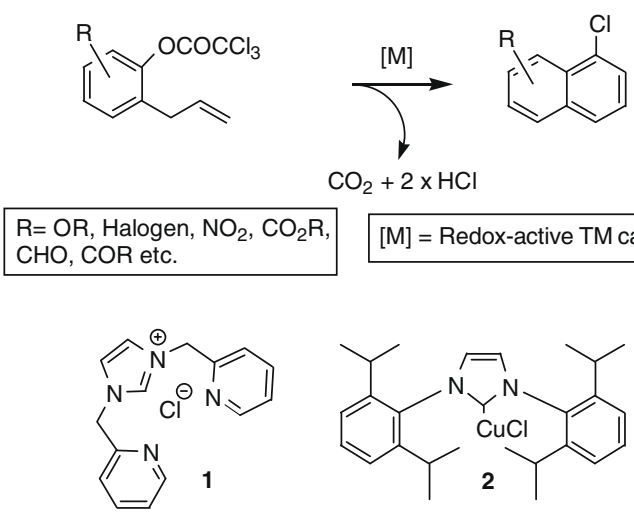

$[\mathrm{M}]=$ Redox-active TM catalyst

Scheme 1. The 'BHQ reaction'. 

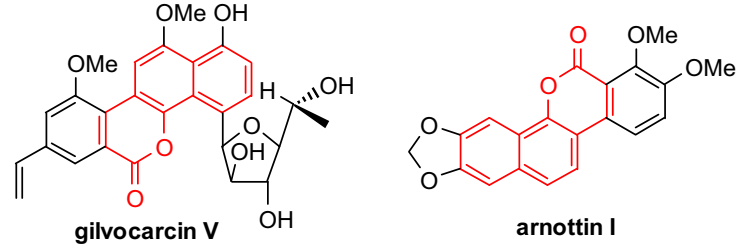

Figure 1. Natural products possessing the $6 H$-benzo[d]naphtha[1,2-b]pyran-6-one ring system.

(pyridine, 1.2 equiv; $\mathrm{Cl}_{3} \mathrm{CCOCl}, 1.2$ equiv; $\mathrm{DCM} ; 0{ }^{\circ} \mathrm{C}$ ) afforded the trichloroacetates $\mathbf{5}$ and $\mathbf{8}$ as crystalline solids which were used without further purification in the crucial BHQ reaction. In the event thermolysis of a $0.23 \mathrm{M}$ solution of $\mathbf{8}$ or $\mathbf{9}$ in DCE (DCE $=1,2$-dichloroethane) containing $\mathrm{CuCl}(5 \mathrm{~mol} \%)$ and the ligand $1(5 \mathrm{~mol} \%)$ for $2 \mathrm{~h}$ at $200{ }^{\circ} \mathrm{C}$ in a microwave reactor ${ }^{11}$ afforded the isomeric benzannulated products ${ }^{12} \mathbf{6}$ and $\mathbf{9}$ in $52 \%$ and $85 \%$ isolated yields, respectively (Scheme 2 ).

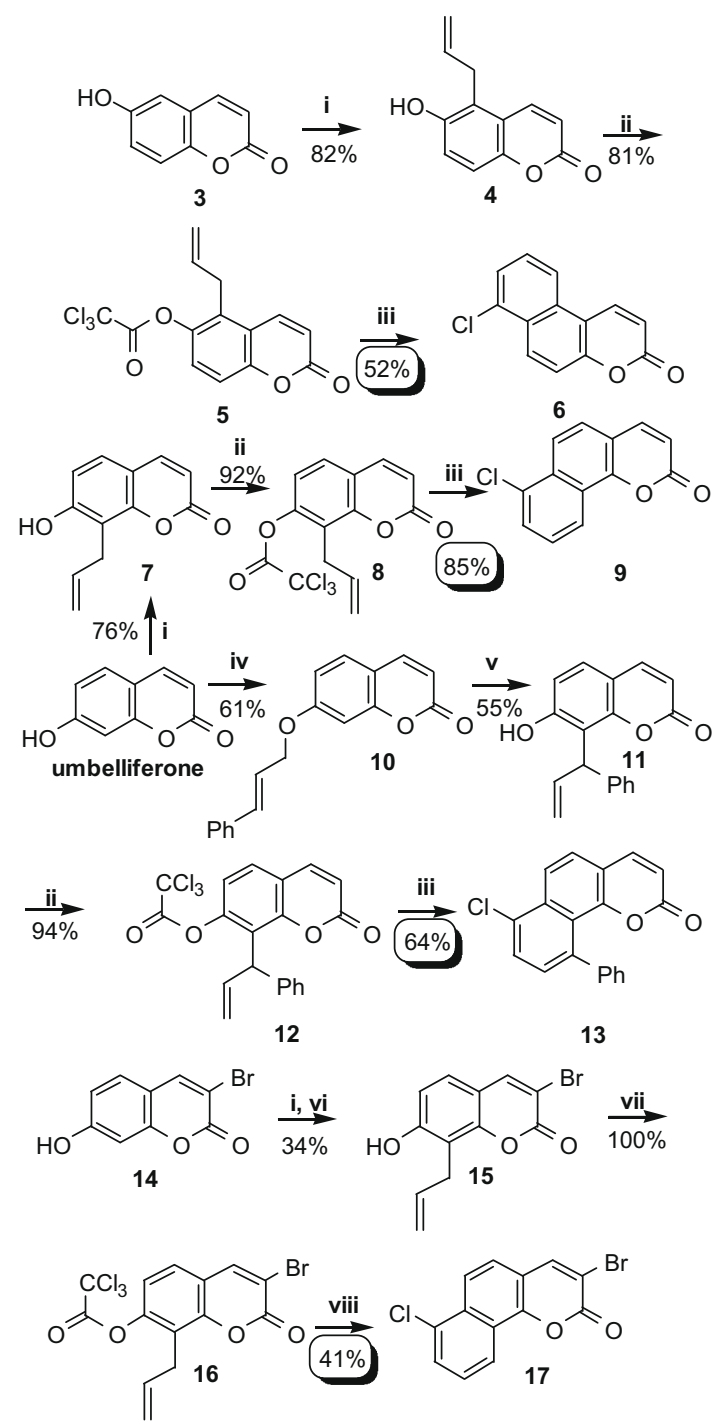

Scheme 2. Initial benzannulation sequences. (i) (a) allyl bromide (1.2 equiv); $\mathrm{K}_{2} \mathrm{CO}_{3}$ (1.2 equiv); acetone; $56{ }^{\circ} \mathrm{C} ; 15 \mathrm{~h}$; (b) for prepn. of 4 : $\mathrm{Ph}_{2} \mathrm{O} ; 260{ }^{\circ} \mathrm{C} ; 1 \mathrm{~h}$; for prepn. 7 : $\mathrm{N}, \mathrm{N}$-diethylaniline; $217^{\circ} \mathrm{C} ; 6 \mathrm{~h}$; (ii) $\mathrm{Cl}_{3} \mathrm{CCOCl}$ ( 1.2 equiv); pyridine (1.2 equiv); $\mathrm{CH}_{2} \mathrm{Cl}_{2} ; 0^{\circ} \mathrm{C} ; 3 \mathrm{~h}$; (iii) 1 (5 mol \%), $\mathrm{CuCl}(5 \mathrm{~mol} \%), \mathrm{DCE}, \mu \mathrm{W}, 200^{\circ} \mathrm{C}, 2 \mathrm{~h}$; (iv) transcinnamyl chloride (1.2 equiv); $\mathrm{K}_{2} \mathrm{CO}_{3}$ (1.2 equiv); acetone; $56{ }^{\circ} \mathrm{C} ; 24 \mathrm{~h}$; (v) $\mathrm{N}, \mathrm{N}$ diethylaniline; $217^{\circ} \mathrm{C} ; 2 \mathrm{~h}$; (vi) toluene, $\mu \mathrm{W}, 220^{\circ} \mathrm{C}, 6 \mathrm{~h}$; (vii) $\mathrm{Cl}_{3} \mathrm{CCOCCl}$ (1.2 equiv); $\mathrm{Et}_{3} \mathrm{~N}$, (1.2 equiv) $\mathrm{Et}_{2} \mathrm{O}, 20^{\circ} \mathrm{C} ; 16 \mathrm{~h}$; (viii) 2 ( $5 \mathrm{~mol} \%$ ); diglyme; $162{ }^{\circ} \mathrm{C} ; 7 \mathrm{~h}$.
A more demanding test of the benzannulation sequence lay in the cyclisation of trichloroacetate $\mathbf{1 2}$, which again was readily available from umbelliferone via the known phenol $11 .{ }^{13}$ On this occasion subjecting 12 to our standard benzannulation conditions again proceeded without incident, affording the sterically congested bi-aryl derivative 13 in good isolated yield (64\% after chromatography). The ability to generate bi-aryls in this manner, without recourse to one of the omnipresent batteries of Pd-mediated coupling strategies now routinely adopted for such transformations, may have broader synthetic scope and is a facet of the BHQ reaction which is currently under further investigation.

The fact that the BHQ reaction is also tolerant of functionality attached to the heterocyclic ring system is exemplified by the benzannulation of trichloroacetate 16, prepared from bromocoumarin $14^{14}$, to the coumarin 17 with moderate efficiency (41\% isolated yield). Parenthetically the major practical drawback of this particular sequence was the capricious nature of the initial Claisen rearrangement. Also of note is the observation that optimal yields of benzannulated product $\mathbf{1 7}$ were, in this case, obtained when the NHC copper complex $\mathbf{2}$ was utilised as the catalyst under purely thermal reaction conditions ${ }^{15}$ (diglyme, $162^{\circ} \mathrm{C}$ ) rather than under conditions of microwave activation as employed previously.

In an attempt to extend the BHQ methodology further, benzannulation of trichloroacetate $\mathbf{1 9}$, a substrate which was readily accessible from the known enol $\mathbf{1 8}^{9}$, was undertaken. Crucially, subjection of the crystalline trichloroacetate $\mathbf{1 9}$ to our standard benzannulation reaction conditions ( $\mathrm{CuCl}$ ( $5 \mathrm{~mol} \%) ; 1$ (5 mol \%); $\mu \mathrm{W} ; 200^{\circ} \mathrm{C} ; 2 \mathrm{~h}$ ) afforded the coumarin 20 in $38 \%$ isolated yield. This result clearly demonstrated, for the first time, that enol trichloroacetates rather than aryl trichloroacetates could also participate in the benzannulation sequence, albeit with a somewhat diminished yield (Scheme 3).

Given that hydrogen chloride is generated during the course of BHQ reactions the synthesis of trichloroacetate $\mathbf{2 3}$ from esculin ${ }^{16}$ was undertaken (Scheme 4). This particular substrate possesses an acid-labile O-glycosidic link whose stability to the relatively harsh conditions of the benzannulation reaction could be monitored with time as the reaction progressed. In this particular instance optimum yields of the coumarin $\mathbf{2 4}$ were realised when a solution of $\mathbf{2 3}$ and carbene complex $\mathbf{2}$ ( $5 \mathrm{~mol} \%$ ) in diglyme was irradiated in a microwave reactor at $170{ }^{\circ} \mathrm{C}$. Examination of the ${ }^{1} \mathrm{H}$ NMR spectrum of the crude reaction mixture from this reaction indicated the presence of only trace quantities of phenol $\mathbf{2 5}$ thereby assuaging our initial fears that acid-labile functionality would be incompatible to the reaction conditions used to promote the benzannulation reaction.

Upon completing these initial methodological investigations we have begun to investigate its application to the synthesis of the aromatic core of the gilvocarcins (Scheme 5). Regioselective 07allylation of esculetin (allyl bromide (1.2 equiv); $\mathrm{K}_{2} \mathrm{CO}_{3}$ ( 1.2 equiv); acetone; $56{ }^{\circ} \mathrm{C} ; 41 \%$ ), followed O6-methylation (71\%) afforded the coumarin 26. ${ }^{17}$ Claisen rearrangement of $\mathbf{2 6}$ was best effected in a microwave reactor ${ }^{13}$ (toluene; $217^{\circ} \mathrm{C}$ ) and was afforded the phenol 27 in essentially quantitative yield after irradiation for $3 \mathrm{~h}$.

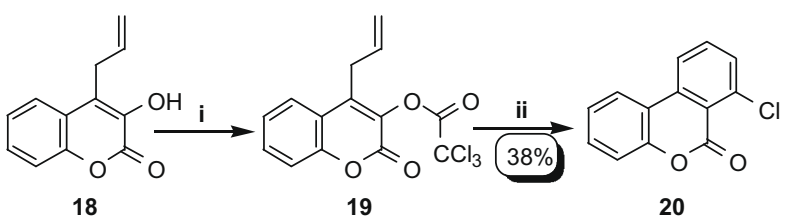

Scheme 3. BHQ reaction using enol trichloroacetates. (i) $\mathrm{Cl}_{3} \mathrm{CCOCCl}$ (1.0 equiv): $\mathrm{Et}_{3} \mathrm{~N}$, (1.0 equiv) $\mathrm{Et}_{2} \mathrm{O}, 20^{\circ} \mathrm{C} ; 16 \mathrm{~h}$; (ii) 1 ( $5 \mathrm{~mol} \%$ ); $\mathrm{CuCl}(5 \mathrm{~mol} \%), \mathrm{DCE} ; \mu \mathrm{W} ; 200{ }^{\circ} \mathrm{C}$; $2 \mathrm{~h}$. 

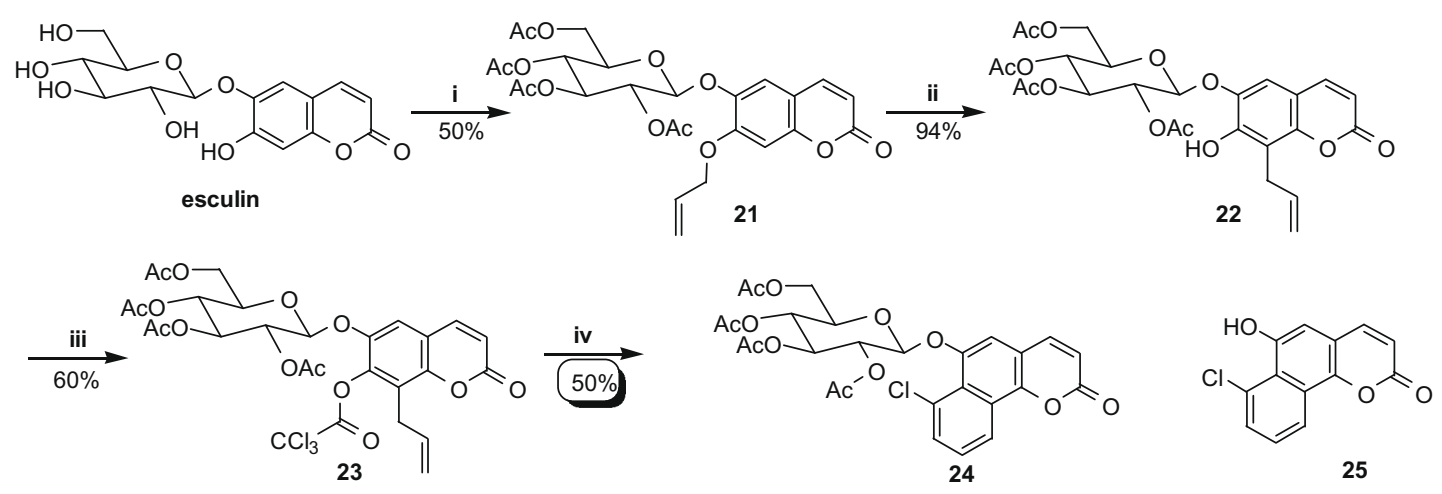

Scheme 4. (i) (a) Allyl bromide (1.2 equiv); $\mathrm{K}_{2} \mathrm{CO}_{3}$ (1.2 equiv); DMF; $90^{\circ} \mathrm{C} ; 7 \mathrm{~h}$; (b) acetic anhydride (1.2 equiv); pyridine, $20^{\circ} \mathrm{C} ; 17 \mathrm{~h}$; (ii) $\mathrm{Ph}_{2} \mathrm{O} ; 210-220^{\circ} \mathrm{C} ; 5 \mathrm{~h}$; (iii) $\mathrm{ClCOCCl}_{3}$, (1.2 equiv); $\mathrm{Et}_{3} \mathrm{~N}$ (1.2 equiv), $\mathrm{Et}_{2} \mathrm{O}, 0{ }^{\circ} \mathrm{C} ; 3 \mathrm{~h}$; (iv) 2 (5 mol \%); diglyme; $\mu \mathrm{W} ; 170{ }^{\circ} \mathrm{C} ; 2 \mathrm{~h}$.

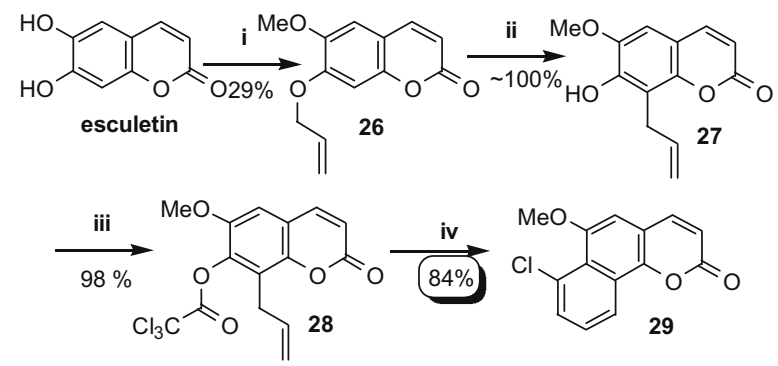

Scheme 5. (i) (a) Allyl bromide ( 1 equiv); $\mathrm{K}_{2} \mathrm{CO}_{3}$ (1 equiv); acetone; $52{ }^{\circ} \mathrm{C} ; 12 \mathrm{~h}$; (b) MeI (1.2 equiv); $\mathrm{K}_{2} \mathrm{CO}_{3}$ (1 equiv); acetone; $20^{\circ} \mathrm{C} ; 15 \mathrm{~h}$; (iii) (a) toluene; $\mu \mathrm{W} ; 217^{\circ} \mathrm{C}$; $3 \mathrm{~h}$; (b) $\mathrm{Cl}_{3} \mathrm{COCl}$ (1.2 equiv); pyridine (1.2 equiv); $\mathrm{DCM} ; 0{ }^{\circ} \mathrm{C}$; (iv) 1 (5 mol \%), $\mathrm{CuCl}$ (5 mol \%); DCE; $\mu \mathrm{W} ; 200{ }^{\circ} \mathrm{C} ; 2 \mathrm{~h}$.

Trichloroacetylation $\left(\mathrm{Cl}_{3} \mathrm{CCOCl}\right.$ ( 1.2 equiv); pyridine (1.2 equiv); $\mathrm{DCM} ; 0^{\circ} \mathrm{C} ; 3 \mathrm{~h} ; 98 \%$ ) of $\mathbf{2 7}$ followed by benzannulation of the crystalline ester $\mathbf{2 8}$ again proceeded smoothly, leading to the isolation of the coumarin $\mathbf{2 9}$ in $84 \%$ after chromatography. With the successful outcome of this study the same sequence was repeated using diol 31 which itself was prepared in 67\% yield via a Pechmann condensation $^{18}$ of $\mathbf{3 0}$ with 2-ethoxycarbonylcyclohexanone. Regioselective etherification (88\%) of $\mathbf{3 1}$ followed by Claisen rearrangement ( $\sim$ quantitative) in a microwave reactor and subsequent trichloroacetylation enabled rapid access to multi-gram quantities of the key intermediate 33 (Scheme 6).

Most gratifyingly subjecting $\mathbf{3 3}$ to our standard benzannulation conditions (1 (5 mol \%); $\mathrm{CuCl}(5 \mathrm{~mol} \%)$, DCE; $\mu \mathrm{W} ; 200{ }^{\circ} \mathrm{C} 2 \mathrm{~h}$ ) resulted in the isolation of $\mathbf{3 4}$ in $92 \%$ yield. Scaling up this reaction with the aid of a CEM MARS Parallel System enabled $6 \mathrm{~g}$ of $\mathbf{3 4}$ to be prepared in a single operation. The regiochemical outcome associated with the initial allylation reaction of $\mathbf{3 1}$ is based upon literature precedent ${ }^{17}$, NOE difference studies on $\mathbf{3 5}$ (Figure 2, Scheme 6) and confirmed by the independent synthesis of $\mathbf{3 2}$ starting from iso-vanillin. ${ }^{19}$ Finally, dehydrogenation of $\mathbf{3 4}$ to the aromatic system 35 was best effected (63\% yield) using $10 \% \mathrm{Pd}-\mathrm{C}$ in refluxing diphenyl ether ${ }^{20}$ under aerobic conditions. Interestingly when the same reaction was conducted under anaerobic conditions methyl ether $\mathbf{3 6}^{21}$, the overall product of a redox process, was isolated in the much reduced yield of $24 \%$. In this case we presume that the 'Pd-H' species generated in the initial dehydrogenation reaction are then responsible for reduction of the $\mathrm{C}-\mathrm{Cl}$ bond in $\mathbf{3 5}$.

In conclusion we have demonstrated that the BHQ benzannulation reaction can be applied to the synthesis of the $6 \mathrm{H}$ benzo[d]naphtha[1,2-b]pyran-6-one ring system. This basic strategy-one which involves the utilisation of simple, yet robust, ortho-Claisen rearrangements followed by ATRC reactions-enables the rapid synthesis of polycyclic coumarin-containing systems, from readily available starting materials. Further application to the synthesis of gilvocarcins is now in hand.

Representative experimental procedure: A solution of trichloroacetate 33 (919 mg, $2.3 \mathrm{mmol}$ ), $\mathrm{CuCl}(5 \mathrm{~mol} \%, 11 \mathrm{mg}, 0.12 \mathrm{mmol}$ ) and ligand 1 ( $5 \mathrm{~mol} \%, 33 \mathrm{mg}, 0.115 \mathrm{mmol}$ ) in freshly distilled 1,2-dichloroethane $(10 \mathrm{~mL})$ was placed in $20 \mathrm{~mL}$ microwave reaction vial and flushed with dry nitrogen. The sealed vial was heated to $200{ }^{\circ} \mathrm{C}$ for $2 \mathrm{~h}$ in a microwave reactor ${ }^{11}$ and then allowed to cool to room temperature. The solvent was removed in vacuo and the residue purified by column chromatography (silica; eluent 1:9 ethyl acetate:petrol) to afford the title compound, $\mathbf{3 4}$ as a yellow

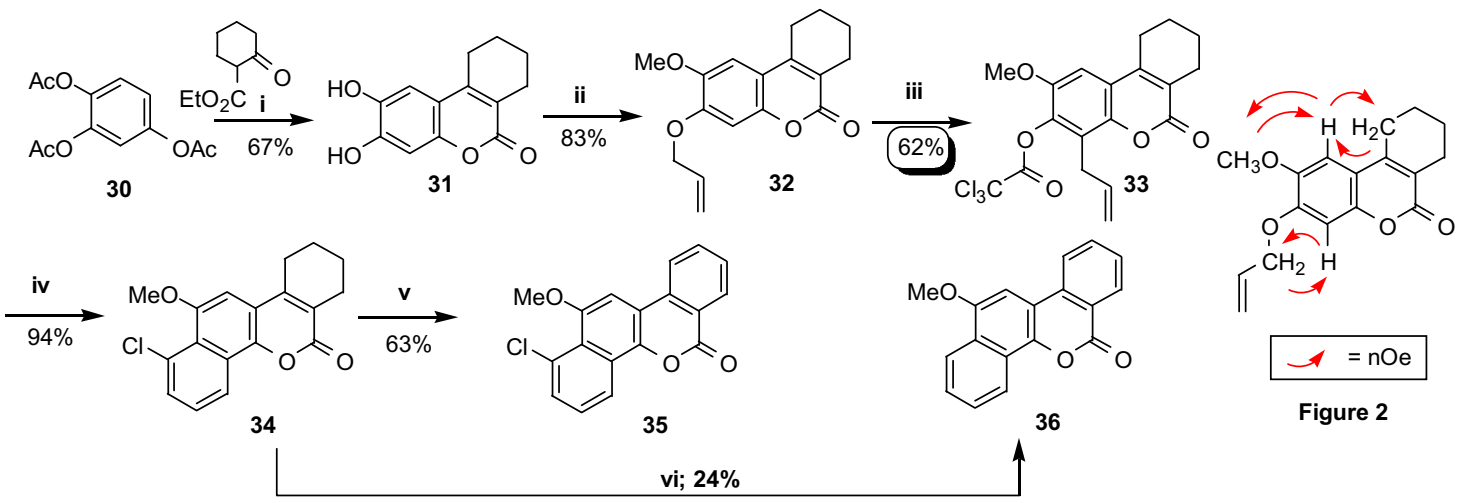

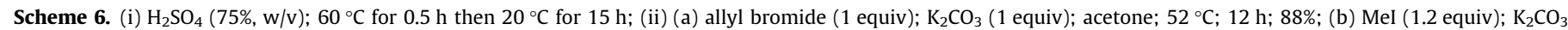

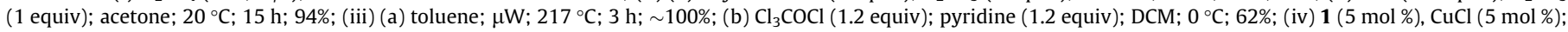
DCE; $\mu \mathrm{W} ; 200^{\circ} \mathrm{C} ; 2 \mathrm{~h}$; (v) $10 \% \mathrm{Pd}-\mathrm{C}$; $\mathrm{Ph}_{2} \mathrm{O} ; 259{ }^{\circ} \mathrm{C}$; in air; $15 \mathrm{~h}$; (vi) $10 \% \mathrm{Pd}-\mathrm{C} ; \mathrm{Ph}_{2} \mathrm{O} ; 259{ }^{\circ} \mathrm{C}$; under $\mathrm{N}_{2} ; 48 \mathrm{~h}$ then at $259{ }^{\circ} \mathrm{C}$ in air; $24 \mathrm{~h}$. 
crystalline solid. Yield $664 \mathrm{mg}$ (92\%); mp $148{ }^{\circ} \mathrm{C} .{ }^{1} \mathrm{H} \quad \mathrm{NMR}$ $\left(500 \mathrm{MHz}, \mathrm{CDCl}_{3}\right) \delta 8.37(\mathrm{~d}, 1 \mathrm{H}, J=8 \mathrm{~Hz}), 7.53(\mathrm{~d}, 1 \mathrm{H}, J=7 \mathrm{~Hz})$, $7.38(\mathrm{t}, 1 \mathrm{H}, J=8 \mathrm{~Hz}), 6.75(\mathrm{~s}, 1 \mathrm{H}), 3.90(\mathrm{~s}, 3 \mathrm{H}), 2.76-2.73(\mathrm{~m}$, $2 \mathrm{H}), 2.58-2.56(\mathrm{~m}, 2 \mathrm{H}), 1.87-1.82(\mathrm{~m}, 2 \mathrm{H}), 1.80-1.75(\mathrm{~m}, 2 \mathrm{H})$ ppm; IR $v_{\max }(\mathrm{KBr}) 2935.1,1705.5,1696.5,1584.9,1570.0$, 1396.1, $112.9 \mathrm{~cm}^{-1}$; MS $\left(\mathrm{EI}^{+}\right) \mathrm{m} / z 299\left(\left[\mathrm{M}-\mathrm{CH}_{3}\right]^{+}, 3 \%\right), 314\left(\mathrm{M}^{+}\right.$, 39\%). $\left(\mathrm{CI}^{+}\right) 315\left([\mathrm{M}+\mathrm{H}]^{+}, 100 \%\right)$; HRMS $\left(\mathrm{EI}^{+}\right) \mathrm{C}_{18} \mathrm{H}_{15} \mathrm{O}_{3} \mathrm{Cl}\left(\mathrm{M}^{+}\right)$requires 314.0698 , found 314.0704 .

\section{Acknowledgement}

J.A.B. and C.L. thank DyStar UK Ltd for support of this research programme.

\section{References and notes}

1. (a) Quayle, P.; Fengas, D.; Richards, S. Synlett 2003, 1797-1800; (b) Edlin, C. D.; Faulkner, J.; Fengas, D.; Knight, C. K.; Parker, J.; Preece, I.; Quayle, P.; Richards, S. N. Synlett 2005, 572-576; (c) Faulkner, J.; Edlin, C. D.; Fengas, D.; Preece, I.; Quayle, P.; Richards, S. N. Tetrahedron Lett. 2005, 46, 2381-2385; (d) Helliwell, M.; Fengas, D.; Knight, C. K.; Parker, J.; Quayle, P.; Raftery, J.; Richards, S. N. Tetrahedron Lett. 2005, 46, 7129-7134; (e) Edlin, C. D.; Faulkner, J.; Quayle, P. Tetrahedron Lett. 2006, 47, 1145-1151; (f) Edlin, C. D.; Faulkner, J.; Helliwell, M.; Knight, C. K.; Parker, J.; Quayle, P.; Raftery, J. Tetrahedron 2006, 62, 30043015; (g) Edlin, C. D.; Faulkner, J.; Fengas, D.; Helliwell, M.; Knight, C. K.; House, D.; Parker, J.; Preece, I.; Quayle, P.; Raftery, J.; Richards, S. N. J. Organomet. Chem. 2006, 691, 5375-5382.

2. Bull, J. A.; Hutchings, M. G.; Quayle, P. Angew. Chem., Int. Ed. 2007, 46, 18691872.

3. Bull, J. A.; Hutchings, M. G.; Lujan, C.; Quayle, P. Tetrahedron Lett. 2008, 49, 1352-1356.

4. Lujan, C. Transfer Report, University of Manchester 2008; Hutchings, M. G.; Quayle, P.; Bull, J. A.; Barroso, C. L. PCT Int. Appl. WO 2007147775 (Chem. Abs. 2008, 148, 402631)

5. For recent reviews see: Diez-Gonzalez, S.; Nolan, S. P. Synlett 2007, 2158-2167; Diez-Gonzalez, S.; Nolan, S. P. Aldrichchim. Acta 2008, 41, 43-51.

6. See: Harrity, J. P. A., Ed.. Tetrahedron 2008, 64, 767-968.

7. For aproaches to this ring system see: Ray, S.; Patra, A.; Mal, D. Tetrahedron 2008, 64, 3253-3267. and refs. therein.

8. (a) Cordero-Vargas, A.; Quiclet-Sire, B.; Zard, S. Z. Org. Biomol. Chem. 2005, 3, 4432-4443; (b) Konno, F.; Ishikawa, T.; Kawaata, M.; Yamaguchi, K. J. Org. Chem. 2006, 71, 9818-9823.

9. Chattopadhyay, S. K.; Neogi, K.; Singha, S. K.; Singha, R. D. Synlett 2008, 11371140.

10. Clarke, D. J.; Robinson, R. S. Tetrahedron 2002, 58, 2831-2837.

11. A Biotage Initiator ${ }^{\mathrm{TM}}$ microwave reactor was used throughout

12. Representative spectroscopic data. 6: ${ }^{1} \mathrm{H}$ NMR $\left(500 \mathrm{MHz}, \mathrm{CDCl}_{3}\right) \delta 8.37-8.34(\mathrm{~m}$, $2 \mathrm{H}), 8.04(\mathrm{~d}, 1 \mathrm{H}, J=8.2 \mathrm{~Hz}), 7.56(\mathrm{~d}, 1 \mathrm{H}, J=7.6 \mathrm{~Hz}), 7.50(\mathrm{t}, 1 \mathrm{H}, J=8 \mathrm{~Hz}), 7.43(\mathrm{~d}$, $1 \mathrm{H}, J=9 \mathrm{~Hz}), 6.52(\mathrm{~d}, 1 \mathrm{H}, J=10 \mathrm{~Hz}) \mathrm{ppm}$; IR $v_{\max }(\mathrm{KBr}) 1732.9,1651.9,1567.8$,
1117.7, 797.0 $\mathrm{cm}^{-1}$; MS (EI $\left.{ }^{+}\right) \mathrm{m} / z 195$ ([M-Cl$\left.]^{+}, 16 \%\right), 230\left(\mathrm{M}^{+}, 100 \%\right)$; HRMS $\left(\mathrm{EI}^{+}\right) \mathrm{C}_{13} \mathrm{H}_{7} \mathrm{O}_{2} \mathrm{Cl}\left(\mathrm{M}^{+}\right)$requires 230.0129 , found 230.0120 . Compound 9: ${ }^{1} \mathrm{H} \mathrm{NMR}$ $\left(500 \mathrm{MHz}, \mathrm{CDCl}_{3}\right) \delta 8.52(\mathrm{~d}, 1 \mathrm{H}, J=9 \mathrm{~Hz}), 8.17(\mathrm{~d}, 1 \mathrm{H}, J=10 \mathrm{~Hz}), 7.89(\mathrm{~d}, 1 \mathrm{H}$, $J=10 \mathrm{~Hz}), 7.77(\mathrm{~d}, 1 \mathrm{H}, J=8 \mathrm{~Hz}), 7.62-7.56(\mathrm{~m}, 2 \mathrm{H}), 6.60(\mathrm{~d}, 1 \mathrm{H}, J=10 \mathrm{~Hz}) \mathrm{ppm}$; IR $v_{\max }(\mathrm{KBr}) 1728.8,1653.2,1602.7,1559.1,1378.2,1330.2,1262.8,1102.4$, 923.0, 837.4, $758.7 \mathrm{~cm}^{-1}$; MS $\left(\mathrm{EI}^{+}\right) \mathrm{m} / z 201$ ([M-COH$\left.]^{+}, 100 \%\right), 229\left([\mathrm{M}-\mathrm{H}]^{+}\right.$, $78 \%$ ); HRMS $\left(\mathrm{EI}^{+}\right) \mathrm{C}_{13} \mathrm{H}_{7} \mathrm{O}_{2} \mathrm{Cl}\left(\mathrm{M}^{+}\right)$requires 230.0129 , found 230.0128 Compound 13: ${ }^{1} \mathrm{H}$ NMR $\left(500 \mathrm{MHz}, \mathrm{CDCl}_{3}\right) \delta 8.19(\mathrm{~d}, 1 \mathrm{H}, J=9 \mathrm{~Hz}), 7.68(\mathrm{t}, 1 \mathrm{H}$, $J=8 \mathrm{~Hz}), 7.52(\mathrm{~d}, 1 \mathrm{H}, J=9 \mathrm{~Hz}), 7.41-7.38(\mathrm{~m}, 3 \mathrm{H}), 7.31(\mathrm{~d}, 1 \mathrm{H}, J=8 \mathrm{~Hz}), 7.28$ $7.26(\mathrm{~m}, 2 \mathrm{H}), 6.30(\mathrm{~d}, 1 \mathrm{H}, J=10 \mathrm{~Hz}) \mathrm{ppm}$; IR $v_{\max }(\mathrm{KBr}) 2364.2,1722.5,1602.6$, 1497.6, 1340.8, 1101.4, 937.6, 877.0 830.9, 699.9 $\mathrm{cm}^{-1}$; $\mathrm{MS}\left(\mathrm{EI}^{+}\right) \mathrm{m} / \mathrm{z} 306\left(\mathrm{M}^{+}\right.$ 100\%); HRMS $\left(\mathrm{ES}^{+}\right) \mathrm{C}_{19} \mathrm{H}_{15} \mathrm{O}_{2} \mathrm{NCl}\left(\left[\mathrm{M}+\mathrm{NH}_{4}\right]^{+}\right)$requires 324.0786, found 324.0781. Compound 17: ${ }^{1} \mathrm{H}$ NMR $\left(300 \mathrm{MHz}, \mathrm{CDCl}_{3}\right) \delta 8.48$ (brd. d, $1 \mathrm{H}$ $J=8 \mathrm{~Hz}) ; 8.24(\mathrm{~s} 1 \mathrm{H}) ; 8.16(\mathrm{~d}, 1 \mathrm{H}, J=9 \mathrm{~Hz}), 7.78(1 \mathrm{H}, \mathrm{dd}, J=8 \mathrm{~Hz}), 7.58(1 \mathrm{H}$, apt $\mathrm{t}, J=8 \mathrm{~Hz}), 7.53(\mathrm{~d}, 1 \mathrm{H}, J=9 \mathrm{~Hz}) \mathrm{ppm}$; IR $v_{\max }(\mathrm{KBr}) 1732.5 \mathrm{~cm}^{-1}$; MS $\left(\mathrm{EI}^{+}\right) \mathrm{m} / z$; HRMS $\left(\right.$ ES $\left.^{+}\right)$. Compound 20: ${ }^{1} \mathrm{H}$ NMR $\left(500 \mathrm{MHz}\right.$, acetone- $\left.d_{6}\right) \delta 8.22(\mathrm{~d}, 1 \mathrm{H}$, $J=8 \mathrm{~Hz}), 8.13(\mathrm{~d}, 1 \mathrm{H}, J=8 \mathrm{~Hz}), 7.71(\mathrm{t}, 1 \mathrm{H}, J=8 \mathrm{~Hz}), 7.57(\mathrm{~d}, 1 \mathrm{H}, J=8 \mathrm{~Hz}), 7.44$ $(\mathrm{t}, 1 \mathrm{H}, J=8 \mathrm{~Hz}), 7.25(\mathrm{t}, 1 \mathrm{H}, J=8 \mathrm{~Hz}), 7.19(\mathrm{~d}, 1 \mathrm{H}, J=8 \mathrm{~Hz}) \mathrm{ppm}$; IR $v_{\max }(\mathrm{KBr})$ $1732.8,1728.8,1447.3,1249.2,1219.1,1045.9,1015.5,804.8,742.1 \mathrm{~cm}^{-1}$; MS $\left(\mathrm{EI}^{+}\right) \mathrm{m} / \mathrm{z} 195$ ([M-C]+1, 3\%), $230\left(\mathrm{M}^{+}, 21 \%\right) \cdot\left(\mathrm{Cl}^{+}\right) \mathrm{m} / \mathrm{z} 214$ ([M-O $\left.]^{+}, 14 \%\right), 231$ $\left([\mathrm{M}+\mathrm{H}]^{+}, \quad 100 \%\right) ;$ HRMS $\left(\mathrm{ES}^{+}\right) \quad \mathrm{C}_{13} \mathrm{H}_{7} \mathrm{O}_{2} \mathrm{Cl}\left(\mathrm{M}^{+}\right)$requires 230.0133, found 230.0129. Compound 24: ${ }^{1} \mathrm{H}$ NMR $\left(500 \mathrm{MHz}, \mathrm{CDCl}_{3}\right) \delta 8.47(1 \mathrm{H}, \mathrm{dd}, J=8$, $J=1 \mathrm{~Hz}), 7.75(1 \mathrm{H}, \mathrm{d}, J=9 \mathrm{~Hz}), 7.68(1 \mathrm{H}, \mathrm{dd}, J=8 \mathrm{~Hz}, J=1 \mathrm{~Hz}), 7.51(1 \mathrm{H}$, apt. t $J=8 \mathrm{~Hz}), 7.13(1 \mathrm{H}, \mathrm{s}), 6.56(1 \mathrm{H}, \mathrm{d}, J=9 \mathrm{~Hz}), 5.48(1 \mathrm{H}, \mathrm{dd}, J=10 \mathrm{~Hz}, J=8 \mathrm{~Hz})$, $5.33(2 \mathrm{H}$, apt. t, $J=9 \mathrm{~Hz}), 5.32(1 \mathrm{H}, \mathrm{d}, J=8 \mathrm{~Hz}), 5.27(1 \mathrm{H}$, apt. t, $J=9 \mathrm{~Hz}), 4.32$ $(1 \mathrm{H}$, apt t $J=12 \mathrm{~Hz}, J=5 \mathrm{~Hz}), 4.24(1 \mathrm{H}, \mathrm{dd}, J=12 \mathrm{~Hz}, J=3 \mathrm{~Hz}), 3.98(1 \mathrm{H}$, ddd, $J=7 \mathrm{~Hz}, J=5 \mathrm{~Hz}, J=3 \mathrm{~Hz}), 2.08\left(3 \mathrm{H}, \mathrm{s}, \mathrm{OCOCH}_{3}\right), 2.06\left(3 \mathrm{H}, \mathrm{s}, \mathrm{OCOCH}_{3}\right), 2.06(6 \mathrm{H}$, $\mathrm{s}, 2 \mathrm{x} \mathrm{OCOCH}$ ) ppm; IR $v_{\max }(\mathrm{film}): 1746.14,1560.2,1458.9,1424.18,1366.6$ 1318.1, 1233.8, 1041.5, $911.7 \mathrm{~cm}^{-1}$; HRMS $\left(\mathrm{ES}^{+}\right) \mathrm{C} 27 \mathrm{H} 25 \mathrm{ClNaO} 12\left([\mathrm{M}+\mathrm{Na}]^{+}\right)$ requires 599.0932; found 599.0942. Compound 25: ${ }^{1} \mathrm{H}$ NMR $\left(500 \mathrm{MHz}, \mathrm{CDCl}_{3}\right)$ $\delta 8.55(1 \mathrm{H}, \mathrm{d}, J=9 \mathrm{~Hz}), 8.02(1 \mathrm{H}, \mathrm{s}), 7.75(1 \mathrm{H}, \mathrm{d}, J=9 \mathrm{~Hz}), 7.61(1 \mathrm{H}, \mathrm{d}, J=8 \mathrm{~Hz})$, $7.53(1 \mathrm{H}$, apt. $\mathrm{t}, J=8 \mathrm{~Hz}), 7.01(1 \mathrm{H}, \mathrm{s}),, 6.56(1 \mathrm{H}, \mathrm{d}, J=9 \mathrm{~Hz}) \mathrm{ppm}$. Compound 29: ${ }^{1} \mathrm{H}$ NMR $\left(500 \mathrm{MHz}, \mathrm{CDCl}_{3}\right) \delta 8.37(\mathrm{~d}, 1 \mathrm{H}, J=9 \mathrm{~Hz}), 7.67(\mathrm{~d}, 1 \mathrm{H}, J=10 \mathrm{~Hz}), 7.59$ $(\mathrm{d}, 1 \mathrm{H}, J=8 \mathrm{~Hz}), 7.42(\mathrm{t}, 1 \mathrm{H}, J=8 \mathrm{~Hz}), 6.69(\mathrm{~s}, 1 \mathrm{H}), 6.47(\mathrm{~d}, 1 \mathrm{H}, J=10 \mathrm{~Hz}), 3.91(\mathrm{~s}$, $\left.3 \mathrm{H}, \mathrm{CH}_{3} \mathrm{O}\right) \mathrm{ppm}$; IR $v_{\max }(\mathrm{KBr}) 2923.7,1715.9,1590.6,1559.8,1379.6,1322.3$ 1123.8, 1103.6, 904.4, 750.0 $\mathrm{cm}^{-1}$; MS $\left(\mathrm{CI}^{+}\right) \mathrm{m} / \mathrm{z} 245\left(\left[\mathrm{M}-\mathrm{CH}_{3}\right]^{+}, 11 \%\right), 260\left(\mathrm{M}^{+}\right.$ $100 \%$ ); HRMS $\left(\mathrm{EI}^{+}\right) \mathrm{C}_{14} \mathrm{H}_{9} \mathrm{O}_{3} \mathrm{Cl}\left(\mathrm{M}^{+}\right)$requires 260.0235 , found 260.0229 .

13. Saidi, M. R.; Rajabi, F. Heterocycles 2001, 55, 1805-1812.

14. Collado, I. G.; Galán, R. H.; Massanet, G. M.; Alonso, M. S. Tetrahedron 1994, 50 6433-6440.

15. The development of 'thermal' BHQ reactions will be detailed elsewhere: Hutchings, M. G.; Luján, C.; Quayle, P. in preparation.

16. For the preparation of $\mathbf{2 1}$ see: Sardari, S.; Mori, Y.; Horita, K.; Micetich, R. G.; Nishibe, S.; Daneshtalab, M. Bioorg. Med. Chem. 1999, 7, 1933-1940.

17. Seshadri, T. R.; Sood, M. S. J. Indian Chem. Soc. 1962, 39, 539-544; See also: Shamis, M.; Barbas, C. F.; Shabat, D. Bioorg. Med. Chem. Lett. 2007, 17, 1172 1175.

18. James, A. L.; Perry, J. D.; Ford, M.; Armstrong, L.; Gould, F. K. J. Appl. Microbiol. 1997, 82, 532-536; James, A; Armstrong, L. PCT Int. Appl. WO 9741138 (Chem. Abs. 1997, 127, 331693).

19. Bull, J. A. Ph.D. Thesis, University of Manchester, 2008.

20. Guillaumet, G.; Hretani, M.; Coudert, G.; Averbeck, D.; Averbeck, S. Eur. J. Med. Chem. 1990, 25, 45-51.

21. Qagaja, G.; Jones, G. B. J. Org. Chem. 2000, 65, 7187-7194. 